\title{
Angiogenesis inhibited by drinking tea
}

Consumption of tea has been shown to $\checkmark$ inhibit the growth of several tumour types in animals, including cancers of the lung and oesophagus ${ }^{1-3}$. Drinking tea, especially green tea, is also associated with a lower incidence of human cancer ${ }^{1}$. The mechanisms of cancer inhibition are not known, although several hypotheses have been proposed. We investigated whether drinking green tea could suppress angiogenesis, a process of blood-vessel growth required for tumour growth and metastasis. We find that green tea, and one of its components, epigallocatechin-3-gallate (EGCG), significantly prevents the growth of new blood vessels in animals. This finding indicates that drinking tea may be beneficial for the prevention and treatment of angiogenesis-dependent diseases, including cancer and blindness caused by diabetes.

EGCG has been reported to inhibit urokinase, which may be used by some tumours for the invasion of neighbouring healthy tissues ${ }^{4}$. However, the effective concentration of EGCG seems to be too high for it to be physiologically relevant for tea

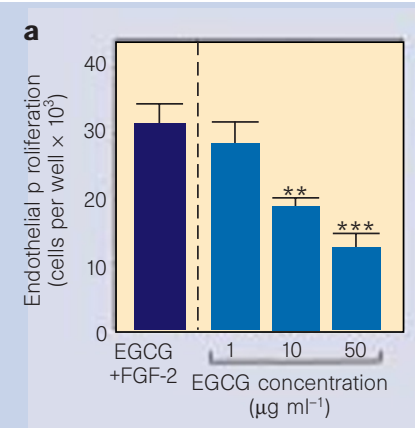

d

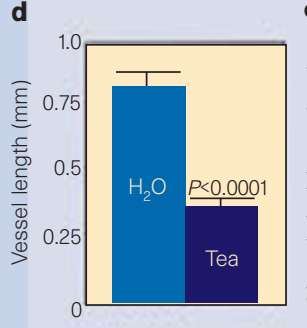

e
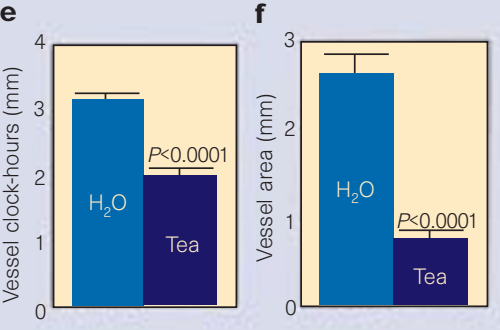

g

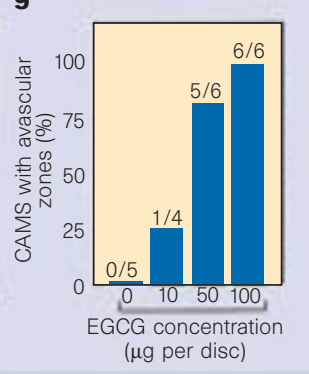

Figure 1 Suppression of endothelial growth and angiogenesis by EGCG and green tea. a, Results of a bovine capillary endothelial cell proliferation assay?. We seeded 10,000 cells per well in gelatinized 24-well plates and grew them in DME medium containing 5\% bovine calf serum. EGCG samples in triplicates and FGF-2 at a final concentration of $1 \mathrm{ng} \mathrm{ml}^{-1}$ were added to each well. After $72 \mathrm{~h}$ incubation, cells were counted with a Coulter counter. Values are mean ( \pm s.e.m.) number of cells from 3 determinations. ${ }^{* *} P<0.005,{ }^{* *} P<0.0001$. b- $\mathbf{f}$, Suppression of VEGF-stimulated vascularization by green tea in the mouse corneal model. Mice drank Chinese green-tea water extract ${ }^{9,10}$ starting at day 3 before VEGF implantation. Pellets containing $160 \mathrm{ng}$ VEGF, sucrose, aluminium sulphate and hydron were implanted into corneal micropockets ${ }^{8}$. $\mathbf{b}$, Cornea of a water-drinking mouse 5 days after implantation. $\mathbf{c}$, Cornea of a green-tea-drinking mouse. $\mathbf{d}$ - $\mathbf{f}$, Vessel length, clock-hours and vessel area. $\mathbf{g}$, Results of the chorioallantoic membrane (CAM) assay ${ }^{8}$. Discs of methylcellulose containing EGCG dried on nylon mesh were implanted on the CAMs of individual embryos. Embryos and CAMs were examined by stereoscope after $48 \mathrm{~h}$ for the formation of avascular zones in the field of the implanted discs. At various doses, the number of CAMs with avascular zones over the total number of CAMs is indicated above each bar. dependent manner (Fig. 1g), as measured by the formation of avascular zones.

To investigate whether tea could suppress angiogenesis, we made green tea the sole drinking fluid for mice and examined the effect of oral consumption on the inhibition of corneal neovascularization stimulated by vascular endothelial growth factor (VEGF). The corneal model is a rigorous antiangiogenic assay, requiring systemic administration of a putative angiogenesis inhibitor to suppress neovascularization induced by $160 \mathrm{ng}$ of VEGF in the cornea. The amount of green tea in the drinking water was $1.25 \%\left(4.69 \mathrm{mg} \mathrm{ml}^{-1}\right)$ containing $708 \mu \mathrm{g} \mathrm{ml}^{-1} \mathrm{EGCG}^{9,10}$. The concentration of EGCG in the plasma was previously reported to be in the range of $0.1-0.3 \mu \mathrm{M}$, which is similar to levels in humans after drinking two or three cups of tea ${ }^{11}$.

This tea preparation has been shown to significantly suppress the growth and progression of tumours in the lungs in animal models ${ }^{9}$. Compared with the control group that drank water alone (Fig. 1b), drinking tea significantly prevented VEGF-induced corneal neovascularization (Fig. 1c). Bloodvessel length (Fig. 1d), clock-hours of corneal neovascularization (the proportion of the circumference that is vascularized if the eye is viewed as a clock; Fig. 1e) and area of neovascularization (Fig. 1f) in seven corneas from four mice in the tea-drinking group were inhibited by approximately 55, 35 and $70 \%$, respectively.

Our data indicate that EGCG suppresses endothelial cell growth in vitro and the formation of new blood vessels in chick chorioallantoic membrane. Drinking green tea significantly prevents corneal neovascularization induced by one of the most potent angiogenic factors, VEGF. Because the growth of all solid tumours is dependent on angiogenesis ${ }^{6}$, this finding may explain why drinking green tea prevents the growth of a variety of different types of tumour.

\section{Yihai Cao, Renhai Cao}

Laboratory of Angiogenesis Research, Microbiology and Tumor Biology Center, Karolinska Institute,

17177 Stockholm, Sweden

e-mail:yihai.cao@mtc.ki.se

\footnotetext{
1. Yang, C. S. \& Wang, Z.-Y. J. Natl Cancer Inst. 85, 1038-1049 (1993). 2. Wang, Z.-Y. et al. Carcinogenesis 16, 2143-2148 (1995)

3. Yang, G.-Y. et al. Cancer Res. 57, 1889-1894 (1997).

4. Jankun, J., Selman, S. H. \& Swiercz, R. Nature 387, 561 (1997).

5. Yang C. S. Nature 389, 134-135 (1997).

6. Folkman, J. Nature Med. 1, 27-31 (1995)

Cao, Y. et al. J. Biol. Chem. 271, 29461-29467 (1996).

8. Cao, Y. et al. J. Exp. Med. 182, 2069-2077 (1995).

9. Yang, C. S. et al. Exp. Lung Res. 24, 629-639 (1998).

10. Wang, Z.-Y. et al. Cancer Res. 52, 1162-1170 (1992).

11. Yang, C. S. et al. Cancer Epidemiol. Biomark. Prev. 7, 351-354 (1998).
} 